\title{
High gain W-shaped microstrip patch antenna
}

\author{
M. N. Shakib ${ }^{1 a)}$, M. Tariqul Islam ${ }^{2}$, and N. Misran ${ }^{1}$ \\ ${ }^{1}$ Department of Electrical, Electronic and Systems Engineering, \\ Universiti Kebangsaan Malaysia (UKM), UKM Bangi, 43600, Selangor, Malaysia \\ ${ }^{2}$ Institute of Space Science (ANGKASA), Universiti Kebangsaan Malaysia (UKM), \\ UKM Bangi, 43600, Selangor, Malaysia \\ a)engmdns@yahoo.com
}

\begin{abstract}
A high gain $\mathrm{W}$-shaped microstrip patch antenna is proposed and experimentally investigated. The patch antenna employs a new inverted $\mathrm{W}$-shaped patch structure with meander probe feed technique. A prototype of the proposed antenna is fabricated and tested for validation. This low-profile antenna is operating for the frequency band of $1.84 \mathrm{GHz}$ to $2.29 \mathrm{GHz}$. It exhibits an impedance bandwidth ( $2: 1 \mathrm{VSWR}$ ) of $21.79 \%$ and a high gain of $10.46 \mathrm{dBi}$ at the frequency of $2.11 \mathrm{GHz}$. The measured result shows the stable radiation characteristics, including a low cross-polarization level below $-20 \mathrm{~dB}$ in both planes.
\end{abstract}

Keywords: patch antenna, wideband antenna, meander probe fed Classification: Electromagnetic theory

\section{References}

[1] W. He, R. Jin, and J. Geng, "E-Shape patch with wideband and circular polarization for millimeter-wave communication," IEEE Trans. Antennas Propag., vol. 56, no. 3, pp. 893-895, 2008.

[2] H. W. Lai and K. M. Luk, "Wideband patch antenna with low crosspolarization," Electron. Lett., vol. 40, pp. 159-160, 2004.

[3] M. T. Islam, M. N. Shakib, and N. Misran, "Modified E-H shaped microstrip patch antenna," IEICE Electron. Express, vol. 6, no. 18, pp. 13501354, 2009.

[4] S. M. Moghadasi, A. R. Attari, and M. M. Mirsalehi, "Wideband lowprofile circular polarized rectanglar loop antenna over a mushroom-like EBG structure," IEICE Electron. Express, vol. 5, no. 15, pp. 550-555, Aug. 2008.

[5] S. H. Wi, Y. B. Sun, I. S. Song, S. H. Choa, I. S. Koh, Y. S. Lee, and J. G. Yook, "Package-Level integrated antennas based on LTCC technology," IEEE Trans. Antennas Propag., vol. 54, no. 8, pp. 2190-2197, 2006.

[6] Z. N. Chen and M. Y. W. Chia, "Broad-band suspended probe-fed plate antenna with low cross-polarization levels," IEEE Trans. Antennas Propag., vol. 51, pp. 345-346, 2003.

[7] R. Chair, C. L. Mak, K. F. Lee, K. M. Luk, and A. A. Kishk, "Miniature wide-band half U-slot and half E-shaped patch antennas," IEEE Trans. Antennas Propag., vol. 53, pp. 2645-2652, 2005. 
[8] C. L. Mak, K. M. Luk, K. F. Lee, and Y. L. Chow, "Experimental study of a microstrip patch antenna with an L-shaped probe," IEEE Trans. Antennas Propag., vol. 48, pp. 777-783, 2000.

\section{Introduction}

The developments of new wireless communication systems in different wireless application have spurred the design of new types of compact, wideband, efficient, high gain and low-cost antennas. Microstrip patch antennas have found extensive application in wireless communication system owing to their advantages such as low-profile, conformability, low-cost fabrication and ease of integration with feed-networks [1]. However, conventional microstrip patch antenna suffers from very narrow bandwidth, typically about $5 \%$ bandwidth with respect to the center frequency. This poses a design challenge for the microstrip antenna designer to meet the broadband techniques $[2,3]$. There are numerous and well-known methods to increase the bandwidth of antennas, including increase of the substrate thickness, the use of a low dielectric substrate, the use of various impedance matching and feeding techniques, the use of multiple resonators, and the use of slot antenna geometry $[4,5]$. However, the bandwidth and the size of an antenna are generally mutually conflicting properties, that is, improvement of one of the characteristics normally results in degradation of the other.

Many papers have addressed the problem of enhancing the bandwidth of the patch antenna. Recently, a patch antenna, which is low in crosspolarization level and wide in impedance bandwidth, designated as the suspended probe-fed plate antenna [6], has been proposed. The antenna has an impedance bandwidth of $20 \%$. However, it was pointed out [6] that using a higher patch height could cause a higher cross-polarization level. And, the antenna gain drops to $5 \mathrm{dBi}$, which is lower than other wide-band patch antennas $[4,5]$. Some other techniques as utilizing the shorting pins or shorting walls on the unequal arms of a U-shaped patch, U-slot patch, or L-probe feed patch antennas, wideband and dual-band impedance bandwidth have been achieved with electrically small size in $[7,8]$. However the achievable gains of these antennas are below $8.5 \mathrm{dBi}$. In addition, these techniques result in high cross-polarization radiation. High cross-polarization not only leads to a distortion in the co-polarization pattern, but also reduces the gain of the antenna. In this paper, a new $\mathrm{W}$-shaped patch with meander probe fed is proposed. A better gain and low cross-polarization level is achieved compare to design reported in $[3,4]$.

\section{Antenna structure}

Fig. 1 depicts the geometry of the proposed patch antenna. In the design, the inverted rectangular patch is supported by a low dielectric superstrate with dielectric permittivity $\varepsilon_{r}$ and thickness $h$. An air-filled substrate is sand- 


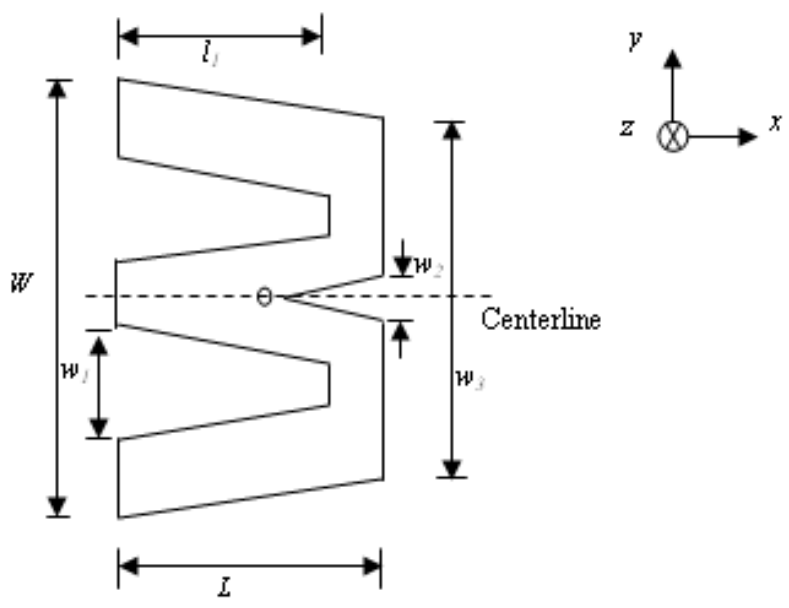

(a)

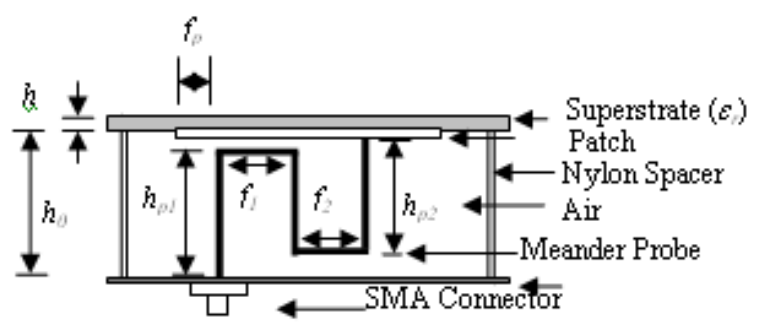

(b)

Fig. 1. Geometry of the proposed patch antenna. (a) Top view and (b) Side view.

wiched between the superstrate and a ground plane. The proposed patch slots are on the same radiating element appear like a $\mathrm{W}$-shaped patch. The proposed patch slots are shown in Fig. 1 (a), where, $l$ and $w$ are the length and width of the slots. The slots (outer widths as $w_{1}$ ) are embedded in parallel on the radiating edge of the patch symmetrically with respect to the centerline ( $x$-axis) of the patch for enhancing the antenna bandwidth. The extra slot (outer width as $w_{2}$ ) is included to reduce the size of the patch. The proposed patch is fed by a meander probe along the centerline ( $x$-axis) of the patch as shown in Fig. 1(b). The optimized design parameters obtained for the proposed patch antenna are $W=76 \mathrm{~mm}, L=50 \mathrm{~mm}, w_{1}=18 \mathrm{~mm}$, $l_{1}=40 \mathrm{~mm}, w_{2}=6 \mathrm{~mm}, w_{3}=66 \mathrm{~mm}, h=1.5748 \mathrm{~mm}, f_{p}=14 \mathrm{~mm}$, $f_{1}=12 \mathrm{~mm}, f_{2}=12 \mathrm{~mm}, h_{p 1}=14 \mathrm{~mm}, h_{p 2}=14 \mathrm{~mm}$. A dielectric substrate with dielectric permittivity, $\varepsilon_{r}$ of 2.2 and thickness, $h$ of 1.5748 has been used in this research. The thickness of the air-filled substrate, $h_{o}$ is $16 \mathrm{~mm}$. An aluminum plate with dimensions of $1.393 \lambda_{0} \times 1.254 \lambda_{0}$ (where $\lambda_{0}$ is the guided wavelength of the centre operating frequency) and thickness of $1 \mathrm{~mm}$ is used as the ground plane. The proposed patch has a simple structure with dimension of $0.516 \lambda_{0} \times 0.38 \lambda_{0}$. The proposed antenna is designed to operate at $1.84 \mathrm{GHz}$ to $2.29 \mathrm{GHz}$ region. In this design, the use of a thick air-filled substrate in between the radiating patch and the ground plane provides the bandwidth enhancement, while the application of superstrate with inverted radiating patch offers a gain enhancement. The use of 
superstrate on the other hand would also provide the necessary protections for the patch from the environmental effects. The use of meander probe fed reduces cross-polarization level. In the design, the vertical portions of the meander probes excite constructively to co-polarization but destructively to cross-polarization. The horizontal portions of the meander probe incorporated with the radiating patch and the ground plane introduce capacitances. These capacitances can suppress some of the inductance contributed by the vertical portions of the probe and hence, achieve low cross-polarization. In addition, the presences of slots also restrict the patch currents, at its resonance frequencies that provide lower resonance on the design. This antenna has wider impedance bandwidth in comparison with the rectangular loop antenna described in [4].

\section{Experimental results}

The resonant properties of the proposed antenna have been predicted and optimized using of a commercial software package HFSS ${ }^{\mathrm{TM}}$. It is measured in an anechoic chamber. Fig. 2 (a) shows the simulated and measured results of the VSWR of the proposed patch antenna. The two closely excited resonant frequencies at $1.88 \mathrm{GHz}$ and at $2.16 \mathrm{GHz}$ as shown in the figure gives the measure of the wideband characteristic of the patch antenna. The measured impedance bandwidth of $21.79 \%$ from $1.84 \mathrm{GHz}$ to $2.29 \mathrm{GHz}$ is achieved at VSWR $\leq 2$ while the simulated patch gives an impedance bandwidth of $24.88 \%(1.83-2.35 \mathrm{GHz})$. The slightly mismatch in between simulated and measured results at second resonant occurs due to fabrication complexity of meander probe feed. The wide-band characteristic is due to large separation between the radiating patch and the ground plane, and due to the use of a low permittivity substrate with the proposed design. The measured gain of the proposed patch antenna at various frequencies is shown in Fig. 2 (b). As shown in the figure, the maximum achievable gain is $10.46 \mathrm{dBi}$ at the frequency of $2.11 \mathrm{GHz}$ and the gain shows stable performance (above $9 \mathrm{dBi}$ ) in the entire operating band. Moreover, the proposed antenna gain is better compare to the designs reported in $[3,4]$. The measured total efficiency of

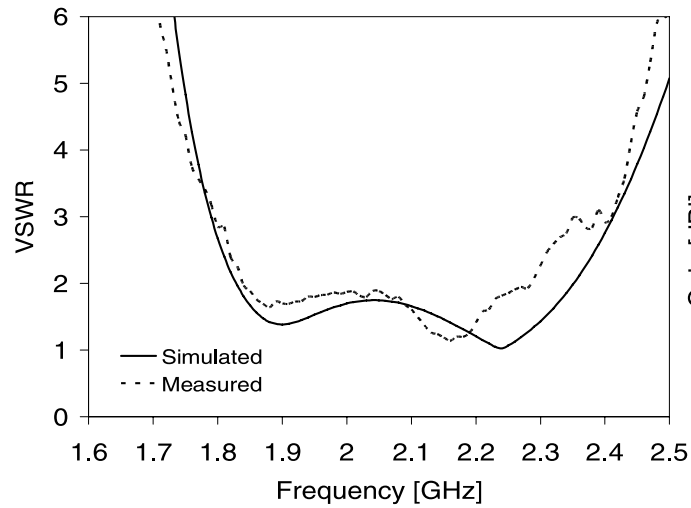

(a)

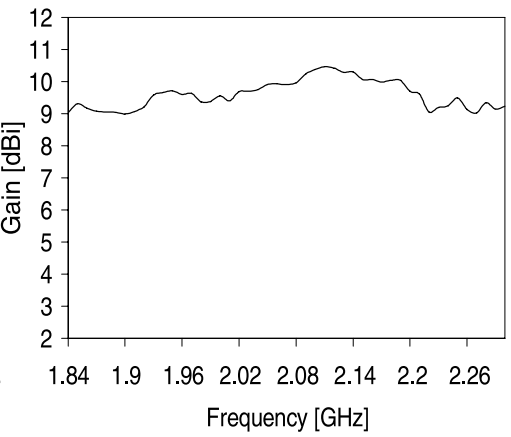

(b)

Fig. 2. (a) VSWR and (b) gain of the proposed antenna. 


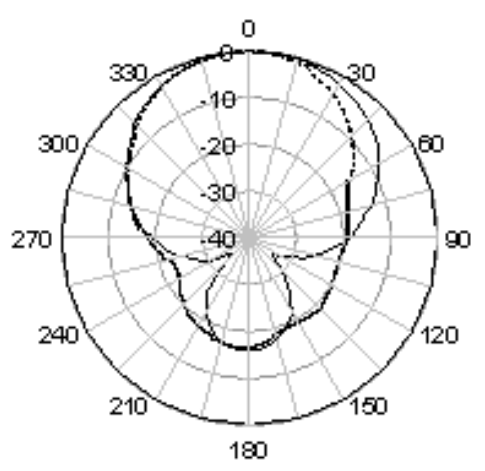

(a)

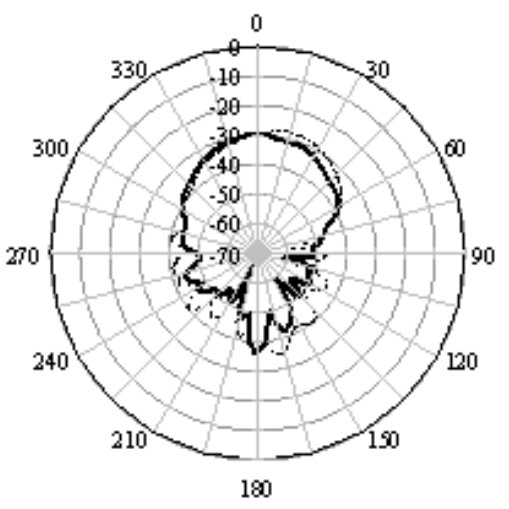

(c)

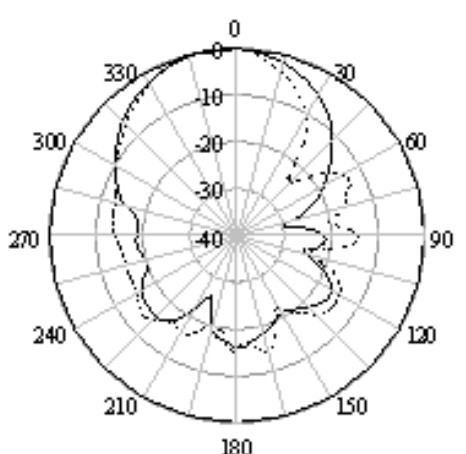

(b)

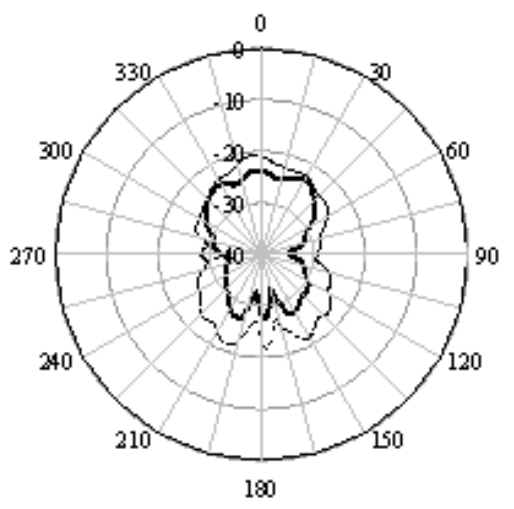

(d)
$-1.88 \mathrm{GHz}, \cdots \cdot 2.16 \mathrm{GHz}$

Fig. 3. Measured radiation pattern in $\mathrm{dB}$ of the proposed antenna. (a) $y z$ co-polar, (b) $x z$ co-polar, (c) $y z$ cross-polar, and (d) $x z$ cross-polar.

the proposed antenna is an average of $90 \%$ over the operational frequency.

Fig. 3 (a) and Fig. 3(b) show the measured radiation patterns of the $y z$-plane and $x z$-plane, respectively. The radiation patterns are measured at resonant frequencies of $1.88 \mathrm{GHz}$ and $2.16 \mathrm{GHz}$. As shown in figure, the designed antenna displays good broadband radiation patterns in the $y z$-plane and $x z$-plane. It can be seen that 3 -dB beamwidth of $66^{\circ}$ and $54^{\circ}$ for $y z$ plane and $x z$-plane respectively at $1.88 \mathrm{GHz}$ and 3 -dB beamwidth of $54^{\circ}$ and $43^{\circ}$ for $y z$-plane and $x z$-plane respectively at $2.16 \mathrm{GHz}$. In Fig. 3 (c) and Fig. 3 (d), the peak cross-polarization level of the antenna is observed to be about $-29 \mathrm{~dB}$ and $-20 \mathrm{~dB}$ below the copolarization level of the main lobe at $y z$-plane and $x z$-plane respectively at the frequency of $1.88 \mathrm{GHz}$. The antenna shows better cross-polarization compare to design reported [3, 4]. It is notable that the radiation characteristics of the proposed patch antenna are better to those of the conventional patch antenna due to good crosspolarization level in both planes are achieved over the impedance bandwidth.

\section{Conclusion}

In this paper, a W-shaped high gain antenna with low cross-polarization level is successfully designed, fabricated and tested. The microstrip patch 
antenna achieves an impedance bandwidth of $21.79 \%$ at VSWR with a maximum gain of $10.46 \mathrm{dBi}$. The measurement results have demonstrated the stability in the radiation performance of the antenna across the entire operating bandwidth. Techniques for microstrip broadbanding, size reduction, and cross-polarization reduction in both planes (below $-20 \mathrm{~dB}$ ) are applied with significant improvement in the design by employing proposed patch design, inverted patch, and meander probe feeding. The proposed antenna with enhanced performance is suitable to be deployed on the base station in an indoor environment.

\section{Acknowledgments}

The authors would like to thank Institute of Space Science (ANGKASA), Universiti Kebangsaan Malaysia (UKM) and the MOSTI Secretariat, Ministry of Science, Technology and Innovation of Malaysia, Science fund: 0101-02-SF0612, for sponsoring this work. 